\title{
Review: mild induced hypothermia does not reduce mortality or severe disability in moderate to severe head injury
}

Alderson P, Gadkary C, Signorini DF. Therapeutic hypothermia for head injury. Cochrane Database Syst Rev 2004;(4):CD001048.

\section{Does mild induced hypothermia reduce mortality and improve long term function in patients with moderate to severe head injury?}

\section{METHODS}

\section{Data sources: Medline, EMBASE/Excerpta Medica, Cochrane Injuries Group Specialised Register, Cochrane Controlled Trials Register (all to 2001); hand searches of conference proceedings and reference lists of relevant trials and review articles; and investigators in the field. \\ Study selection and assessment: randomised controlled trials (RCTs) that compared mild therapeutic hypothermia (local or systemic therapeutic cooling [using a fluid filled cooling blanket, a "Bear Hugger" air cooling device, ice water lavage, or combination, or other method] to a target temperature $\leqslant 34$ $35^{\circ} \mathrm{C}$ for $\geqslant 12$ hours beginning on admission to the intensive care unit or when intracranial pressure [ICP] became uncontrollable by conventional management) with control (open or normothermia) in patients with any closed head injury requiring hospital admission. Individual study quality was assessed based on allocation concealment and blinding of outcome assessors. \\ Outcomes: all cause mortality and death or severe disability (Glasgow Outcome Scale score of severe disability or persistent vegetative state or equivalent measure). Secondary outcomes were complications (pneumonia, coagulopathy, and other serious adverse events) and mean ICP during treatment.}

\section{MAIN RESULTS}

14 RCTs $(\mathrm{n}=1094)$ met the selection criteria. Allocation was concealed in 5 RCTs and unclear in 9 RCTs; outcome assessment was blinded in 6 RCTs and unblinded or unclear in 8 RCTs. Metaanalysis using a fixed effects model showed that immediate induced hypothermia and normothermia did not differ for all cause mortality (odds ratio [OR] $0.80,95 \%$ CI 0.61 to 1.04 ), and death or severe disability (OR 0.75 , CI 0.56 to 1.00 ) (table). More patients who received hypothermia developed pneumonia than those who received normothermia (OR 1.95, CI 1.18 to 3.23). None of the studies reported on any of the other secondary outcomes. I RCT $(n=33)$ compared deferred hypothermia with normothermia. The groups did not differ for all cause mortality (OR 0.21 , CI 0.04 to 1.05) or risk of pneumonia (OR 1.13, CI 0.18 to 6.93). The deferred hypothermia group had a reduced risk of death or severe disability compared with the normothermia group (OR 0.10, CI 0.01 to 1.00 ).

For correspondence: Dr P Alderson, National Institute for Clinical Excellence, London, UK. phil.alderson@nice.nhs.uk

Source of funding: no external funding.

\section{CONCLUSION}

Mild induced hypothermia does not reduce mortality or severe disability in patients with moderate to severe head injury and is associated with an increased risk of pneumonia.

\section{Commentary}

Trentir he review by Alderson et al integrates the findings of new studies to estimate the effects of mild induced hypothermia on patients with brain injuries, building on 2 previous systematic reviews. ${ }^{1} 2$ The findings of Alderson et al were counter to those of an earlier iteration of this review and another cautiously positive review, although similar to those of another recent review. ${ }^{2}$

The trials included by Alderson et al varied on key methodological elements such as allocation concealment and blinding of outcome assessment, as well as clinical features. Such variation can influence estimation of effects, and the reviewers therefore conducted analyses to explore the effect of heterogeneity. These analyses were unable to explain which elements might be responsible for the variation. Trials that investigated immediate hypothermia were analysed separately from 1 trial that investigated deferred hypothermia. The substantially reduced risk of death or severe disability reported in this single small trial merits further research.

This review does not rule out the possibility of smaller effects than a sample of 1000 with a control event rate of approximately $30 \%$ could detect. Additionally, the results can vary depending on which studies are included and how they are pooled in the analysis. Arguably, there is a non-significant trend towards benefit with induced hypothermia. However, the results are unlikely to confer enough of a benefit to outweigh the substantial risk of developing pneumonia.

In light of the absence of clear benefit, the practice of routine therapeutic hypothermia for clinical management of traumatic brain injury should be reconsidered where currently implemented. Current evidence does not support the clinical use of induced hypothermia, except within the context of further research. Further studies are being done with subgroups of patients with brain injury, such as those with hypothermia. However, new regimens for induced hypothermia should not precede the publication of the findings of these trials.

Blair Donkin, RN, BN, CCNS Dunedin Hospital Dunedin, New Zealand

Mclntyre LA, Fergusson DA, Hébert PC, et al. Prolonged therapeutic
hypothermia after traumatic brain injury in adults: a systematic review. hypothermia after traumatic brain injury in adults: a systematic review. JAMA 2003;289:2992-9.

2 Henderson WR, Dhingra VK, Chittock DR, ef al. Hypothermia in the management of traumatic brain injury. A systematic review and metaanalysis. Intensive Care Med 2003;29:1637-44.

\begin{tabular}{|c|c|c|c|c|c|}
\hline \multirow[b]{2}{*}{ Outcomes at 3-12 months } & \multirow[b]{2}{*}{ Number of trials ( $n$ ) } & \multicolumn{2}{|c|}{ Weighted event rates } & \multirow[b]{2}{*}{ RRR $(95 \% \mathrm{Cl})$} & \multirow[b]{2}{*}{ NNT (Cl) } \\
\hline & & Hypothermia & Normothermia & & \\
\hline \multirow[t]{2}{*}{ Death or severe disability } & $\begin{array}{l}12(1061) \\
9(746)\end{array}$ & $\begin{array}{l}28 \% \\
50 \%\end{array}$ & $\begin{array}{l}33 \% \\
57 \%\end{array}$ & $\begin{array}{l}14 \%(-3 \text { to } 28) \\
12 \%(0 \text { to } 23)\end{array}$ & $\begin{array}{l}\text { Not significant } \\
\text { Not significant }\end{array}$ \\
\hline & & & & RRI (Cl) & NNH (Cl) \\
\hline Pneumonia & $7(281)$ & $44 \%$ & $29 \%$ & $51 \%(10$ to 108$)$ & 7 (4 to 25$)$ \\
\hline
\end{tabular}

